NASA Technical Memorandum 83713

\title{
Spin Analysis of Concentrated Traction Contacts
}

Stuart H. Loewenthal

Lewis Research Center

Cleveland, Ohio

Prepared for the

Fourth International Power Transmission and Gearing Conference sponsored by the American Society of Mechanical Engineers Cambridge, Massachusetts, October 8-12, 1984 


\title{
SPIN ANALYSIS OF CONCENTRATED TRACTION CONTACTS
}

Stuart H. Loewenthal

\author{
National Aeronautics and Space Administration \\ Lewis Research Center \\ Cleveland, Ohio 44135
}

\begin{abstract}
Spin, the result of a mismatch in contact radii on either side of the point of rolling, has a detrimental effect on traction contact performance. It occurs in concentrated contacts having conical or contoured rolling elements, such as those in traction drives or angular contact bearings, and is responsible for an increase in contact heating and power loss. This investigation examines the kinematics of spin producing contact geometries and the subsequent effect on traction and power loss. The influence of lubricant traction characteristics and contact geometries that minimize spin are also addressed.
\end{abstract}

\section{INTRODUCTION}

In the contact analysis of many machine components, such as bearings, gears, cams and traction drives, the traction forces and resultant power loss due to sliding and rolling is of engineering importance. The effective traction forces generated in the contact dictate the amount of slip occurring in ball bearings, the skew in roller bearings and the creep rate across a traction-drive contact. The power loss generated in the contact is not only important in and of itself but also dictates the operating temperature. This, in turn, influences the quality of lubrication in the contact and its ultimate durability.

In view of this, the production of traction contact performance and motion has been the subject of numerous investigations through the years [1-9]. Poritsky, et al. [1] and Reichenbach [2] analyzed the frictional 
spinning moment of angular-contact ball bearings. They assumed that relative slip occurred at all points within the contact between the balls and raceways due to a rigid body rotation about a normal axis through the contact center. This special case of spin about an axis located in the center of the contact is often referred to as "pivoting". Wernitz [3] and Magi [4] investigated the more general case of a variable-speed traction drive contact where the slip due to torque transfer caused the spin axis or "spin pole" to move away from the contact center.

Johnson [5] examined the displacement, motion and surface tractions of an unlubricated, free rolling (1.e., without applied tangential forces) ball against a plate under pivoting spin. In contrast to the previous investigations [ 1 to 4], Johnson theoretically showed that at least for small spin velocities, the elastic tangential compliances of the surfaces would enable the central region of the contact to experience no slip, i.e., "locked", while slip occurred only in a region at the perimeter of the contact. The boundary between the "locked" and "slip" region is dictated by the locus of points at which the local value of tangential traction equals or exceeds the limiting value. Johnson's [5] theoretical and experimental work alșo revealed that a. ball rolling while spinning could develop a transverse force causing it to follow a curve trajectory, not unlike a billiard ball that has been struck off center. A more refined theory is given by Kalker [6].

Later work by Johnson $[7,8]$, for lubricated contacts clearly established the visco-elastic behavior of the elastohydrodynamic ofl film where the same type of side thrust can also be developed under spin due to the elasticity of the film at small strain rates. Graphical solutions of the Johnson model presented by Tevaarwerk in [9] permit direct evaluation and design optimization of traction drive contacts. 
Poon [10] and Lingard [11] also developed methods to predict the available traction forces of a contact experiencing spin. Poon's method [10] uti1ized traction data from a twin disk tester together with contact kinematics to predict the available traction. Lingard [11] provided a theoretical approach in which the EHD film exhibited Newtonian viscous behavior at low shear rates until a critical limiting shear stress was reached. This same model was successfully employed by Gaggermeier [12] in a comprehensive investigation of the losses and characteristics of traction drive contacts.

In most of the aforementioned investigations, the knowledge of the angular spin velocity $\omega_{s}$ of the contact is required before detalled calculations of contact performance can be undertaken. For contacting bodies of varying geometry whose axis of rotation are not parallel, the magnitude of $\omega_{s}$ is not readily apparent. Furthermore, means to alter contact geometry in order to minimize $\omega_{s}$ and thus improve performance is often difficult to visualize. It therefore became a basic objective of the current investigation to study the effects of contact geometry on the magnitude of $\omega_{s}$ and its subsequent effects on traction and power loss.

\section{NOMENCLATURE}

a

b

c

E

e

$F_{x}, F$ $\mathrm{J}_{1} \rightarrow \mathrm{J}_{7}$ contact ellipse semi-width in y-direction (transverse to direction of rolling), $m$

contact ellipse semi-width in x-direction (rolling), $m$

lubricant contact parameter defined in Eq. (18) coefficients

modulus of elasticity, GPa

spin pole offset defined in Eq. (1), m

traction forces in $x$ and $y$ direction, $N$

dimensionless traction contact parameters defined in Eqs. (11)

to (17) 


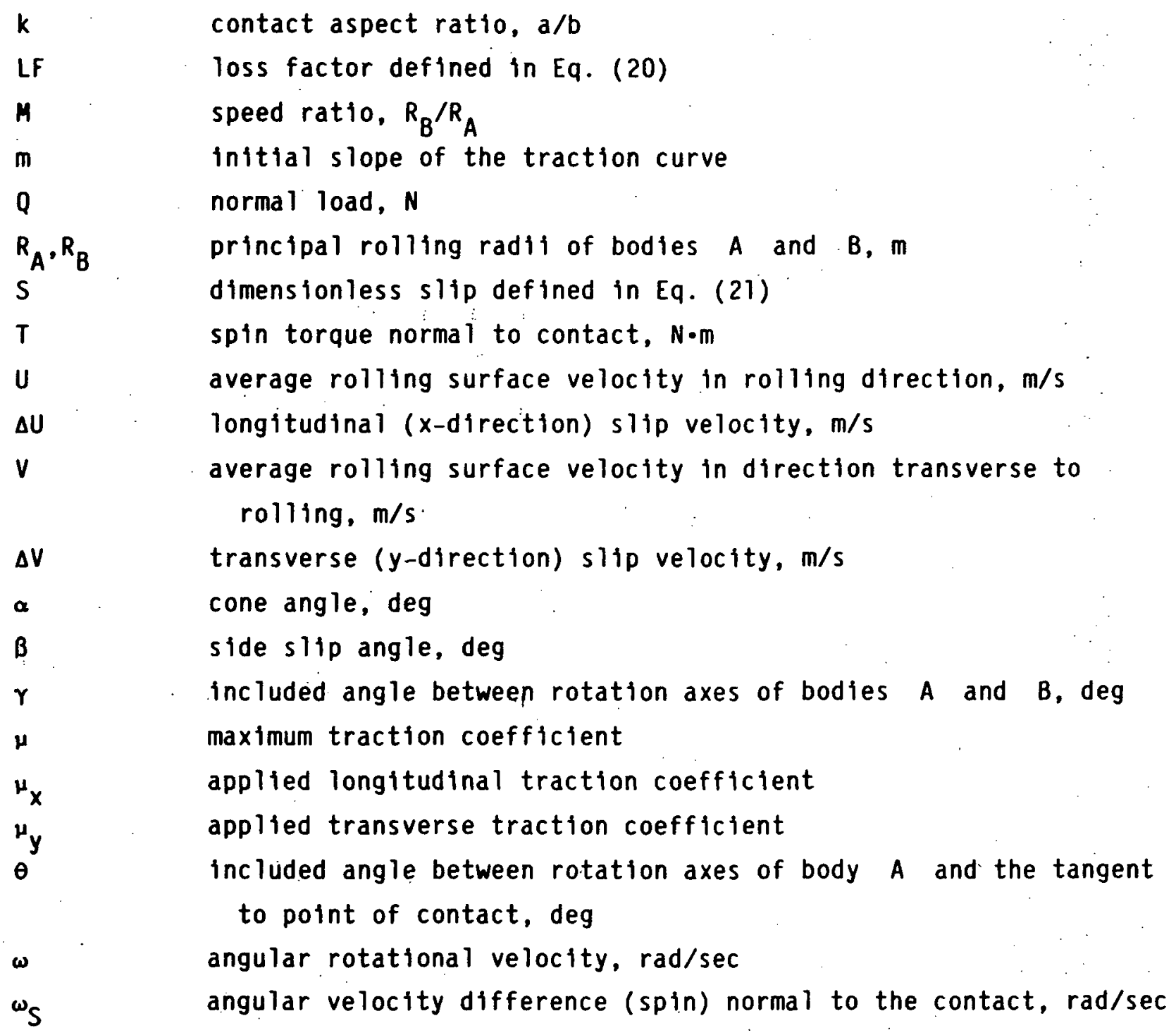

Subscripts
$A, B$
contacting bodies $A$ and $B$
n
normal to the contact
$t$ tangent to the contact
$x, y$
direction with rolling and transverse to rolling

\section{TRACTION CONTACT FORCES}

Before a detailed traction contact performance analysis can be performed, the overall magnitude and direction of the local slip velocities within the contact must be determined. It is these local slip velocities which are responsible for the traction force patterns appearing in Fig. 1 . This figure 
shows the distribution of local traction vectors in the contact when longitudinal traction, misalignment, and spin are present. These traction forces will align themseives with the local slip velocities. In tractiondrive contact some combination of traction, misalignment, and spin are always present. Their effects can be simply superimposed. To determine the performance of a traction-drive contact, the elemental traction forces must be integrated over the contact area.

Because of the parabolic pressure distribution, the elemental traction forces are largest near the center of the contact and diminish in magnitude near the contact perimeter as fllustrated in Fig. 1. As expected, in the case of longitudinal traction, (Fig. $1(a)$ ), the forces align themselves in the rolling direction. With the addition of misalignment (Fig. 1(b)), a sidesip velocity is introduced causing the vectors to cock in line with the sideslip angle. Using conical rollers generally results in a circumferential slip pattern referred to as spin ( $F i g . \quad(C)$ ). This rotary motion is due to the fact that the contact is in pure rolling only at its center. At the right hand edge of the contact the upper roller is sliding over the lower roller because of the mismatch in contact radil. At the left hand edge the situation is reversed and so is the direction of sip. Spin can be thought of as a difference in the angular velocity vector between the bodies in the direction normal to the contact.

Spin generally occurs in traction drives with conical rollers or rollers with nonparallel rotation axes. It is inevitably present in all variable ratio drives at least one or more positions of operation and also in angular ball bearings. The spin velocity causes a loss in power and also reduces the avallable traction coefficient. Furthermore, contact spin can generate appreciable side forces similar to those that cause a spinning ball to roll along a curved path as demonstrated by Johnson [5]. Unlike side slip, both 
the magnitude and direction of spin changes across the contact, with steadily increasing spin velocities moving away from the center of the contact. However, the angular spin velocity $\omega_{S}$ remains constant about a line normal to the contact. When no external torque is applied, this line joins the geometric centers of the driving and driven roller pair as illustrated in fig. $I(c)$. When an external torque is applied, longitudinal slip $\Delta U$ develops which causes the axis of spin to move transversely some distance e (see Fig. 2). This distance $e$ has special meaning in traction contact analysis $[3,4]$. It is referred to as the spin pole offset and is given by the relation:

$$
\mathbf{e}=\frac{\Delta U}{\omega_{S}}
$$

The power transferred between rollers is determined from a summation of the traction force components aligned in the rolling direction times their respective rolling velocities. It is clear from Fig. I that in misalignment only a portion of the traction force is generating useful traction and that the remainder is generating useless side force. For pure spin $(e=0)$ no useful traction is developed, since the elemental traction forces cancel one another. Since the contact power loss is proportional to the product of the elemental traction forces and slip velocities, the presence of spin and misalignment adversely affects efficiency. Furthermore, both conditions lower the avaliable traction coefficient. This reduces the amount of torque that can be safely transmitted. This loss in traction capacity is due to the fact that slip will occur when the local sliding velocities in the contact, exceed the sliding velocity corresponding to the point of peak traction. As an example, take the case of a traction contact with a rolling velocity $U$ of 100 $\mathrm{m} / \mathrm{s}$ and a longitudinal slip velocity $\Delta U$ of a $1 \mathrm{~m} / \mathrm{s}$ at the point of gross sliding. If this contact is misaligned by just $0.5 \mathrm{deg}$, a side-sip velocity $\Delta V=U \tan B=0.87 \mathrm{~m} / \mathrm{s}$ would be generated. Since gross sliding will occur if 
the combined slip velocity reaches: $1 \mathrm{~m} / \mathrm{s}$ anywhere in the contact, the longitudinal slip component $\Delta U$ will be limited to $0.49 \mathrm{~m} / \mathrm{s}$ or less. At the point of impending gross slip, the applied traction force in the rolling direction w1ll be 49 percent of the original non-misaligned value and the induced side ways thrust due to misalignment will be nearly 1.8 times larger than that in the rolling direction. This simple example underscores the need to maintain precise alignment between power transmitting rollers.

\section{CONTACT KINEMATICS UNDER SPIN}

A sample of adjustable speed traction drive geometries appears in Fig. 3. Some of these configurations were in use at the turn of the century [13]. Most of those pictured are still commercially avallable and perform speed matching service for light duty factory equipment.

The degree of spin generated in the contact of traction drives such as those shown in Fig. 3 can be analyzed using a simple model. Consider the general contact geometry of a roller pair rolling without an applied tangential force as illustrated in Fig. 4. The angular spin velocity $\omega_{S, A}$ acting on body $A$ is given by the velocity diagram to be equal to the angular velocity difference normal to the contact, that is:

$$
\vec{\omega}_{S, A}=\vec{\omega}_{B, N}-\vec{\omega}_{A, N}
$$

or in terms of the operating angles $\gamma$ and $\theta$ :

$$
\omega_{S, A}=\omega_{A} \sin \theta-\omega_{B} \sin (\gamma-\theta)
$$

Where $\omega_{A}$ and $\omega_{B}$ are the angular rotational velocities of bodies $A$ and $B, \gamma$ is the included angle between the axes of rotation and $\theta$ is the included angle between body $A^{\prime}$ 's axis of rotation and the tangent to the point of contact. Note $\omega_{A}$ and $\omega_{B}$ are taken to have the rotational direction as shown in Fig. 4 and spin $\omega_{S, A}$ is taken to have a negative value in the clockwise direction. 
Since the velocity pattern on body $B$ is opposite to that of body $A$, it follows that:

$$
\vec{\omega}_{S, B}=-\vec{\omega}_{S, A}
$$

Reversing the direction of rotation will simply cause a "sign" change of the terms in the above equations.

The angular spin velocity can also be found from the difference in the surface velocity distribution between the bodies divided by the distance from the point of pure rolling as shown in $\mathrm{Fig} .4(\mathrm{a})$. At the perimeter of the contact this leads to

$$
\vec{\omega}_{S, A}=\frac{\vec{v}_{A}-\vec{v}_{B}}{a}=\omega_{A}\left(R_{A}+a \sin \theta\right)-\omega_{B}\left[R_{B}+a \sin (\gamma-\theta)\right]
$$

Noting that at the point of rolling, $\omega_{A} R_{A}=\omega_{B} R_{B}$, and simplifying, Eq.

(5) leads immediateiy to the result given in Eq. (3).

\section{Effect of Geometry}

The effects of variation in roller geometry on $\omega_{S}$ can be conveniently studied by "normalizing" Eq. (3) by $\omega_{A}$ as follows:

$$
\frac{\omega_{S, A}}{\omega_{A}}=\sin \theta-\frac{1}{M} \sin (\gamma-\theta)
$$

where $M=$ speed ratio $=\omega_{A} / \omega_{B}=R_{B} / R_{A}$

In the case of a variable ratio traction drive, the rolling radif of one or both bodies and/or spin angles $\gamma$ and $\theta$ will change with a change in speed ratto. Eq. (6) can be used to compute the amount of spin at each position. This is shown graphically in Fig. 5 for the case of equal sized rollers $M=1$. It is instructive to note from setting Eq. (6) to zero that there are two conditions which result in zero spin. These are

$$
Y=\theta=0
$$


and

$$
M=\frac{R_{B}}{R_{A}}=\frac{\sin (\gamma-\theta)}{\sin \theta}
$$

The first condition is the trivial solution which is the case of two parallel, crowned cylinders. The second condition corresponds to the case where the tangent to the point of contact intersects the point where the rotation axes of bodies $A$ and $B$ cross. This is the case of the tapered bearing or bevel gear: Although variable speed drives can be constructed to achieve a condition of zero spin at one or more points, it is impractical or at least extremely difficult to satisfy either of the above conditions at all intermediate positions of operation. Nevertheless, the ratio schedule described by Eq. (8) can serve as a target to minimize spin.

Spin maxima and minima can readily be found by differentiating Eq. (6) with respect to $\theta$ and setting this value to zero. This yields

$$
\theta=\tan ^{-1}\left[-\frac{M+\cos \gamma}{\sin \gamma}\right]
$$

Substituting values of $\theta$ from Eq. (9) for a given $M$ into Eq. (6) will give the minima (most negative) spin values when $r$ is positive while negative $\gamma$ will provide the spin maxima (most positive values). Complementary angles $\left(\theta+180^{\circ}\right)$ or $\left(\theta-180^{\circ}\right)$ will provide equal magnitude but opposite sense spin values. It is instructive to note that the geometry that produces the greatest spin is for $\theta= \pm 90^{\circ}$ and $Y=0$. This corresponds to the case of two parallel, but offset axes disks making face contact as illustrated in Fig. 5 at $\theta=90^{\circ}$. The maximum magnitude of spin at this condition is:

$$
\frac{\omega_{S, A}}{\omega_{A}}= \pm\left(1+\frac{1}{M}\right)
$$


This peak spin ratio $=2$ at $M=1$ and steadily decreases with an increase in speed ratio $M$. The magnitude of the spin ratio at any intermediate combination of geometry angles $Y$ and $\theta$ will never exceed that given by Eq. (10).

Heathcoate slip - The relationships given above are applicable to both external and internal counterformal contacts, that is those having positive transverse radil of curvature. The analysis is also applicable to conformal contacts provided that the difference in transverse curvature is relatively large. However, the analysis needs to be modified for contacts that are closely conformal such as that between a ball and a raceway in a ball bearing. This is because a ball rolling in a closely conforming groove will have two, not just one point of pure rolling and two spin poles will be established. A circular spin pattern will be set up around each spin pole, not unlike Fig. $1(c)$, of equal magnitude but opposite direction. This phenomena, first observed by Heathcoate [14], is addressed in greater detail in [15].

Spin geometries - A wide variety of spin producing geometries with positive and negative spin angles can be devised as illustrated in Fig. 6 . However, not all combinations of $\gamma$ and $\theta$ will produce workable solutions for $Y>90$ or $Y<-90^{\circ}$. In practice, mechanical interference can occur at some points due to the finite width of the rollers. The "sign" of the angles shown in Fig. 6 are applicable to all of the previous equations. In general, geometries in which the crossing point of body B's rotation axis and the common contact tangent, lie on the same side of body $A, i . e ., Y$ and $\theta$ have the same "sign", produce less spin than when the crossing points straddle body A. 


\section{PERFORMANCE ANALYSIS UNDER SPIN}

As mentioned earlier there have been many investigations concerning the prediction of traction in EHO contacts [7 to 12]. One of the more recent and more comprehensive traction contact models is that due to Johnson and Tevaarwerk [8].

Their model covers the full range of viscous, elastic, and plastic behavior of the EHD film. This type of behavior depends on the Deborah number, a relative measure of elastic to inelastic response, and the strain rate. At low pressures and speeds (low Deborah number), the film exhibits linear viscous behavior at low strain rates. It becomes increasingly more nonlinear with increasing strain rate. At higher pressures and speeds, more typical of traction orive contacts, the response is linear and elastic at low rates of strain. At sufficiently high strain rates, the shear stress reaches some limiting value and the film shears plastically as in the case of some of the earlier traction analytical models.

In [9 and 16], Tevaarwerk presents graphical solutions developed from the Johnson and Tevaarwerk elastic-plastic, isothermal traction model. These solutions are of practical value in the design and optimization of traction drive contacts. By knowing the initial slope (related to the shear modulus) and the maximum traction coefficient (related to the limiting shear stress) from a zero spin/zero-side-silp traction curve, the traction, creep, spin torque, and contact power loss can be found over a wide range of spin values and contact geometries.

These solutions are general in nature and provide a good basis for estimating the performance of a traction contact of a known geometry. These solutions will be repeated here in part without theoretical justification. Those interested in the theoretical basis of the Johnson and Tevaarwerk analysis should consult $[8,9$ and 16$]$. 
Dimensionless Traction Parameters

In the Johnson and Tevaarwerk. model several dimensionless parameters were identified that best generalized the results of their analysis. These parameters can be written in terms of the shear modulus and 1 imiting shear stress properties of the lubricant or in terms of the measured initial slope $m$ and peak traction coefficient $\mu$ from a simple experimental traction curve. It is more convenient and probably more rellable at present to work with actual traction data rather than fundamental fluid property data. Fluid property data are usually generated under experimental conditions that are much different than in a traction contact. For the Johnson and Tevaarwerk dimensionless groupings, slope and traction coefficient data must be obtained from a zero side-siip/zero-spin traction curve for the lubricant in question. This reference data must also be obtained at the same contact pressure, temperature, rolling speed and for the same aspect ratio, area and disk material as the contact to be analyzed. However, approximate compliance corrections to the slope can be made if the aspect ratio and contact area of the reference data is different than that of the contact to be evaluated (see [17]).

With the Johnson and Tevaarwerk analysis, knowing fust $m$ and $\mu$ from a simple traction test leads to the prediction of the entire traction-creep curve in the isothermal region under any combination of side-slip and spin. Also, the traction force perpendicular to the rolling direction and contact power losses can be readily determined. Traction data useful for design purposes of two common traction fluids over a wide range of operating conditions appears in [17].

The solutions to this analysis are given in terms of the following dimensionless parameters: 


$$
\begin{aligned}
& \text { silp, } \quad J_{1}=c \frac{\Delta U}{U} \\
& \text { side slip, } \quad J_{2}=C \frac{\Delta V}{U} \\
& \text { spin, } \quad J_{3}=c \frac{\omega_{S} \sqrt{a b}}{U} \\
& \text { traction, } \quad J_{4}=\frac{{ }^{\mu} x}{\mu} \\
& \text { side traction, } \quad J_{5}=\frac{\mu_{y}}{\mu} \\
& \text { torque normal } \quad J_{6}=\frac{T}{\mu Q \sqrt{a b}} \\
& \text { total power } \\
& \text { loss, } \\
& \mathrm{J}_{7}=\mathrm{J}_{4} \mathrm{~J}_{1}+\mathrm{J}_{5} \mathrm{~J}_{2}+\mathrm{J}_{6} \mathrm{~J}_{3} \\
& =\frac{C}{\mu Q U}\left(F_{x} \Delta U+F_{y} \Delta V+T \omega_{S}\right)
\end{aligned}
$$

where $C=$ lubricant contact parameter $=\frac{3 \pi}{8} \frac{m}{\mu} \sqrt{k}$

The spin term $J_{3}$ can be quickly found from the basic geometry of the roller pair with the aid of Eq. (6). Noting that $U=\omega_{A} R_{A}=\omega_{B} R_{B}$, this gives:

$$
J_{3}=c \sqrt{a b}\left[\frac{\sin \theta}{R_{A}}-\frac{\sin (\gamma-\theta)}{R_{B}}\right]
$$

It is important to note that the detrimental effects of spin increase with the square root of contact area (proportional to $\sqrt{a b}$ ). Thus an increase in normal load for a given geometry will also increase $J_{3}$. Furthermore, all other things being equal, highly conformal contacts will be more spin sensitive than those which are not. 
The power loss term $J_{7}$ can be put in a more convenient form in terms of a loss factor LF where

$$
L F=\frac{J_{7}}{J_{4}}=C \quad\left(\frac{\text { Power Loss }}{\text { Power Input }}\right)
$$

Effects of Lubricant on Traction Under Spin

In [17] the traction characteristics of two modern traction fluids were modeled based on traction disk machine test data. A finding of this study was that lubricant composition has a significant effect on traction performance under spin as fllustrated in Fig. 7 from [17]. This figure shows that the degradation of maximum traction coefficient with spin was significantly greater for one of the test fluids. However, under conditions of low spin, this fluid offers comparable or slightly better performance.

\section{Effect of Sip and Spin on Traction}

The graphical solutions appearing in [16] can be used to predict the influence of slip, side-sip and spin on the traction in both the $x$ and $y$ directions. These are shown in the figures which follow. Fig. 8 shows the theoretical effects of slip and spin at a contact aspect ratio of $k=1$. This figure is, in effect, a theoretical traction curve. It is apparent from Fig. 8 that spin tends to increase slip at a given level of traction $J_{4}$, except at low spin values $\left(J_{3}\right.$ less than about 0.7$)$. At low spin values most of the contact is being strained elastically (energy is recoverable) so there is little adverse effects.

If no spin is present and there is no side-silip, a simple relation exists between traction $J_{4}$ and sip, $J_{1}$ of the form:

$$
J_{4}=\frac{2}{\pi}\left[\tan ^{-1} s+\frac{S}{1+s^{2}}\right]
$$


where

$$
S=\frac{\pi}{4}\left(\frac{m}{\mu}\right) \frac{\Delta U}{U}
$$

It may be observed from Eq. (21) that contacts with small value of aspect ratio $k$, hence larger slip values $s$, tend to improve traction. The traction in the contact is directiy related to the total lubricant strain. Since low aspect ratio contacts have longer contact lengths in the direction of rolling they permit a higher bulld up strain, hence more traction.

Under the condition of pure spin, that is no slip, the total strain on the fluid film in the contact transverse to the rolling direction steadily increases in the inlet region, reaches a maxima, and then steadily decays to zero at the outlet of the contact. This accumulated axial strain gives rise to a side traction $J_{5}$ which can be a significant fraction of the maximum traction coefficient, particularly for contacts with low aspect ratio, $k$. The maximum value of sideways traction $\mathrm{J}_{5}$ under pure spin conditions is shown in Fig. 9. Side thrust forces under pure spin can be greater than 60 percent of the limiting traction force. At a given aspect ratio, the magnitude of $J_{5}$ will increase with spin, reach a maxima and then decrease for increasing spin as elastic effects diminish. This can be seen from fig. 10 for $k=1$. Increasing slip in the rolling direction has a relatively minor effect on side traction $J_{5}$ until the slip reaches some value where elastic effects are negligible. At this point there is a precipitous drop in the sideways traction.

The thrust direction with which this sideways traction $J_{5}$ acts is dependent on the geometry of the contact and its direction of rotation. Referring to Fig. 4, the sideways traction acting on the body in the plane of contact, is oriented in the same direction as the bodies angular rotational velocity vector for positive $\omega_{S}$ (CCW spin) and in the opposite direction for negative $\omega_{S}$ (CW spin) for $-90<\gamma<90^{\circ}$. 
It is clear from Eqs. (14) and (15) that multiplying the computed value of $\mathrm{J}_{4}$ and $\mathrm{J}_{5}$ by the maximum available traction coefficient will give the appropriate values of $\mu_{x}$ and $\mu_{y}$ at any slip, side-siip, or spin condition. If spin is present, it is likely that thermal heating will cause some reduction in the value of $\mu$. Using the value of $\mu$ under the spin condition present, if such data is known, to correct $J_{4}$ and $J_{5}$ will yield the most accurate results. Analytical methods to account for thermal effects in the film can be found in [18 to 20$]$.

\section{Traction Contact Power Loss}

Ignoring the rolling traction loss for the moment, the losses in a traction contact of three components: longitudinal slip, side-siip and spin as given by Eq. (17). By dividing the dimensionless contact loss term $\mathrm{J}_{7}$ to the longitudinal traction. term $J_{4}$ we can establish the loss factor LF given in Eq. (20). Power loss then is simply:

$$
\text { Power loss }=\left(\frac{L F}{C}\right) \text { Power input }
$$

In most traction drives a mechanism is used to automatically adjust the normal load in direct proportion to the transmitted force. This forces the traction coefficient in the contact $\mu_{x}$ to be a constant. The geometry of the loading mechanism is selected to be some fraction, typically 70 to 80 percent, of the maximum traction coefficient available (1.e., $J_{4}=0.7$ to 0.8) under the most unfavorable operating condition. This is to provide some safety margin from slipping.

To fllustrate the effect that spin has on power loss, the theoretical loss factor is plotted in Fig. 11 against $J_{3}$ for a constant loading fraction $J_{4}=0.75$ at various $k$ values. It can be observed that imposed.spin has little or no influence on the loss factor LF at low values of spin. 
The losses in the contact at low spin are basically those due to the creep normally associated with torque transfer. From a design point of view, values of spin $J_{3}$ up to approximately 1 can be allowed without 111 effects. At higher levels of spin, above $J_{3} 6$, LF increases directly with spin $J_{3}$ as the lubricant film in the contact shears plastically [9]. Thermal effects then become increasingly more pronounced and the isothermal loss curve in Fig. 10 starts requiring thermal modifications [18 to 20].

The relative power loss between contacts with low spin $\left(\mathrm{J}_{3}<1\right)$ and high spin $\left(\mathrm{J}_{3}>10\right)$ can be better appreciated by cross plotting Fig. 11 against contact aspect (ellipse) ratio. This is done in Fig. 12. High spin contacts have losses that are about an order of magnitude higher than low spin contacts. Reducing contact ellipse ratio $k$ improves efficiency at both low and high levels of spin. However, at high spin levels, the minimum losses occur at $k=1$ and reducing $k$ further, i.e., orienting the ellipse's major axes with the direction of rolling, will cause an increase in power loss.

It is instructive to note that a reduction in $k$, that is, making the contact more circular in shape, will improve efficiency. It will also, however, have a detrimental effect on fatigue life. This is illustrated in Fig. 12 where relative fatigue life from the analys is published in [21] and relative power loss found in [9] is plotted against $k$ for constant torque, normal load, size and traction coefficient. Note that decreasing $k$ by decreasing the relative transverse radius of curvature at constant normal load will cause the contact area to also decrease. This in turn will increase the contact stress, but lessen the nondimensional spin term $J_{3}$ due to the $\sqrt{a b}$ term in Eq. (19).

It should also be mentioned that the contact losses of the Johnson and Tevaarwerk method outlined here are only those associated with traction torque 
transfer. However, while these losses are obviously significant, they are not the only losses to be considered when assessing the performance of a traction drive. Some traction drive designs require large support bearings through which some or all of the clamping loads between roller components must pass. The power losses associated with these bearings can be as great or greater than the contact losses. In fact, in Gaggermeier's experimental investigation [12] with the Arter type toroidal drive, the load-dependent bearing losses were comparable to the contact losses due to power transfer. The drive idling (no-load) losses were as large or larger than both of these load dependent losses combined. These idling losses include oil splashing losses, air windage, seal drag and those losses in the traction rollers and bearings which are independent of transmitted load.

\section{SUMMARY OF RESULTS}

A kinematic analysis of spin producing contacts has been performed. The analysis relates the geometry of the roller pair contact, defined by the intersection of the axes of rotation and tangent to the point of contact, to. the magnitude of spin generated. A solution for the ideal, zero spin producing geometry was given. The effect of spin on traction, creep and power loss was investigated using the Johnson and Tevaarwerk isothermal traction model. The influence of lubricant characteristics on spin traction was also discussed. The effect of contact aspect ratio on relative power loss and fatigue life under spin was presented.

The following findings were obtained:

1. A rolling contact will have zero spin if the rolling radif ratio $R_{B} / R_{A}=\sin (\gamma-\theta) / \sin \theta$. This is the condition where the tangent to the point of contact and both bodies' rotation axes mutually intersect as in the case of a tapered bearing. 
2. The maximum magnitude of spin occurs for $\theta= \pm 90^{\circ}$ and $\gamma=0$.

This corresponds to the case of two parallel, but offset axes disks making face contact.

3. Increases in slip and power loss due to spin are negligible for small amounts of spin $\left(\mathrm{J}_{3}\right.$ less than about 1$)$. Beyond this threshold, slip and power loss increase rapidly with spin as inelastic shearing of the lubricant film occurs.

4. Sideways thrust forces which are greater than 60 percent of the limiting traction force can be generated for bodies rolling under pure spin.

5. The adverse effects of spin can be minimized by reducing the contact aspect ratio $k$ but at the expense of higher contact stresses and lower contact fatigue life.

\section{REFERENCES}

1. Poritsky, H., Hewlett, C. W., Jr., and Coleman, R. E., Jr., "Sliding Friction of Ball Bearings of the Pivot Type," Journal of Applied Mechanics, V. 14, No. 4, Dec. 1947, pp. A261-A268.

2. Reichenbach; G. S., "The Importance of Spinning Friction in ThrustCarrying Ball Bearings," Journal of Basic Engineering, V. 82, June 1960, pp. $295-301$.

3. Wernitz, W., "Friction at Hertzian Contact with Combined Roll and Twist," Rolling Contact Phenomena, Bidwe11, J.B., ed., Elsevier, New York, 1962, pp. 132-1567.

4. Magi, M., "On Efficiencies of Mechanical Coplanar Shaft Power Transmissions" Chalmers University of Technology, Gothenburg, Sweden, 1974.

5. Johnson, K. L., "The Effect of Spin Upon Rolling Motion of an Elastic Sphere on a Plane," Journal of Applied Mechanics, V. 25, No. 3, Sept. 1958, pp. 332-338.

6. Kalker, J. J., "Rolling with Slip and Spin in the Presence of Dry Friction," Wear, V. 9, 1966, pp. 20-38.

7. Johnson, K. L. and Roberts, A. D., "Observations of Viscoelastic Behavior of an Elastohydrodynamic Lubricant $F 11 \mathrm{~m}$, "Proceedings of the Royal

Soctety of London, Series A, V. 337, No. 1609, Mar. 19, 1974, pp. 217-242. 
8. Johnson, K. L. and Tevaarwerk, J. L., "Shear Behavior of Elastohydrodynamic 011 Films," Proceedings of the Royal Society of London, Series A. V. 356, No. 1685, August 1977, pp. 215-236.

9. Tevaarwerk, J. L., "Traction Drive Performance Prediction for the Johnson and Tevaarwerk Traction Model," NASA TP-1530, 1979.

10. Poon, S. Y., "Some Calculations to Assess the Effect of Spin on the Tractive Capacity of Rolling Contact Drives," Proceedings of the Institution of Mechanical Engineers, V. $1851970 / 71$, pp. 1015-1022.

11. Lingard, S., "Tractions at the Spinning Point Contacts of a Variable Ratio Friction Drive," Tribology Intenational, V. 7, No. 5, Oct. 1974, pp. 228-234.

12. Gaggermeier, Helmut, "Investigations of Tractive Force Transmission in Variable Traction Drives in the Area of Elastohydrodynamic Lubrication," Ph.D. Dissertation, Technical University of Munich Germany, 1977.

13. Loewentha 1, S. H., Anderson, N. E. and Rohn, D. A., "Advances in Traction Drive Technology," SAE Paper No. 831304, Sept. 1983.

14. Heathcote, H. L., Proc. Inst. Automobile Engrs. London, V. 15, 1921, p. 569.

15. Harris, T. A., Rolling Bearing Analysis, Wiley, New York, 1966.

16. Tevaarwerk, J. L. and Johnson, K. L., "The Influence of Flutd Rheology on the Performance of Traction Drives," Journal of Lubrication Technology, v. 101, No: 3, July 1979, pp. 266-274.

17. Loewenthal, S. H. and Rohn, D. A., "Elastic Model of the Tractive Behavior of Two Traction Lubricants," ASLE Transactions, V. 27, No. 2, Apr. 1984, pp. 129-137.

18. Johnson, K. L. and Greenwood, J. A., "Thermal Analysis of an Eyring Fluid in Elastohydrodynamic Traction," Wear, V. 61, 1980, pp. 353-374.

19. Tevaarwerk, J. L., "A Simple Thermal Correction for Large Spin Traction Curves," Journal of Mechanical Design, V. 103, No. 2, Apr. 1981, pp. 440-446.

20. Tevaarwerk, J. L., "Traction in Lubricated Contacts," presented at the International Symposium on Contact Mechanics and Wear of Ra11/Wheel Systems, (Vancouver, B.C.), July 6-9, 1982.

21. Rohn, D. A., Loewentha 1, S. H. and Coy, J. J., "Simplified Fatigue Life Analys is for Traetion Drive Contacts," Journal of Mechanical Design, $V$. 103, No. 2, Apr. 1981, pp. 430-439. 


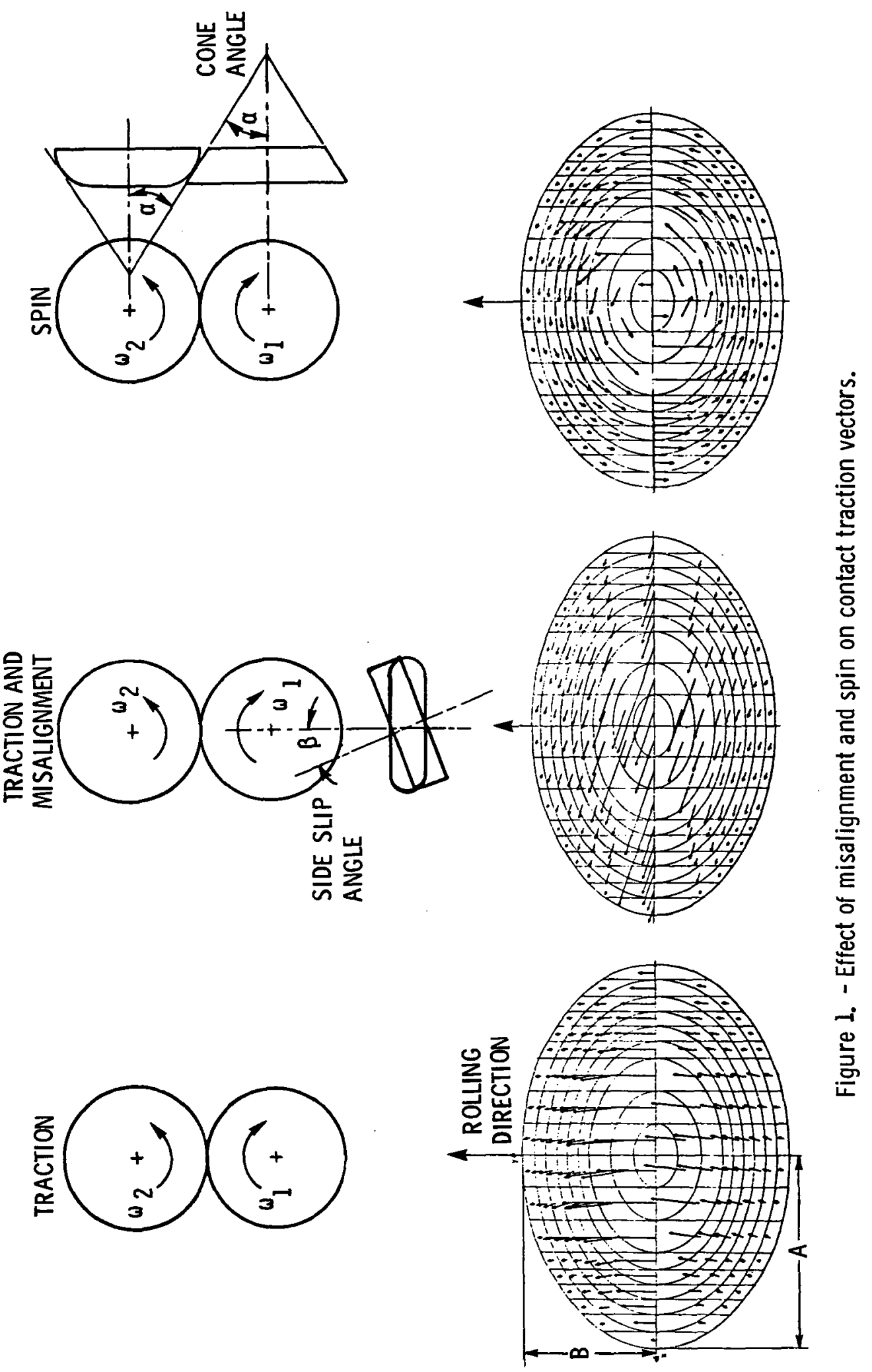




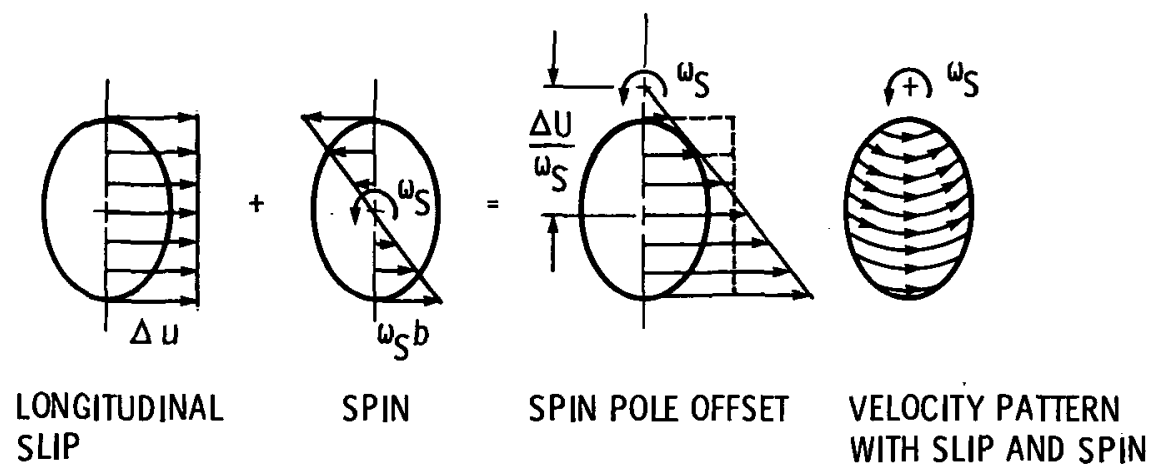

Figure 2. - Contact velocity diagram for combined slip and spin.
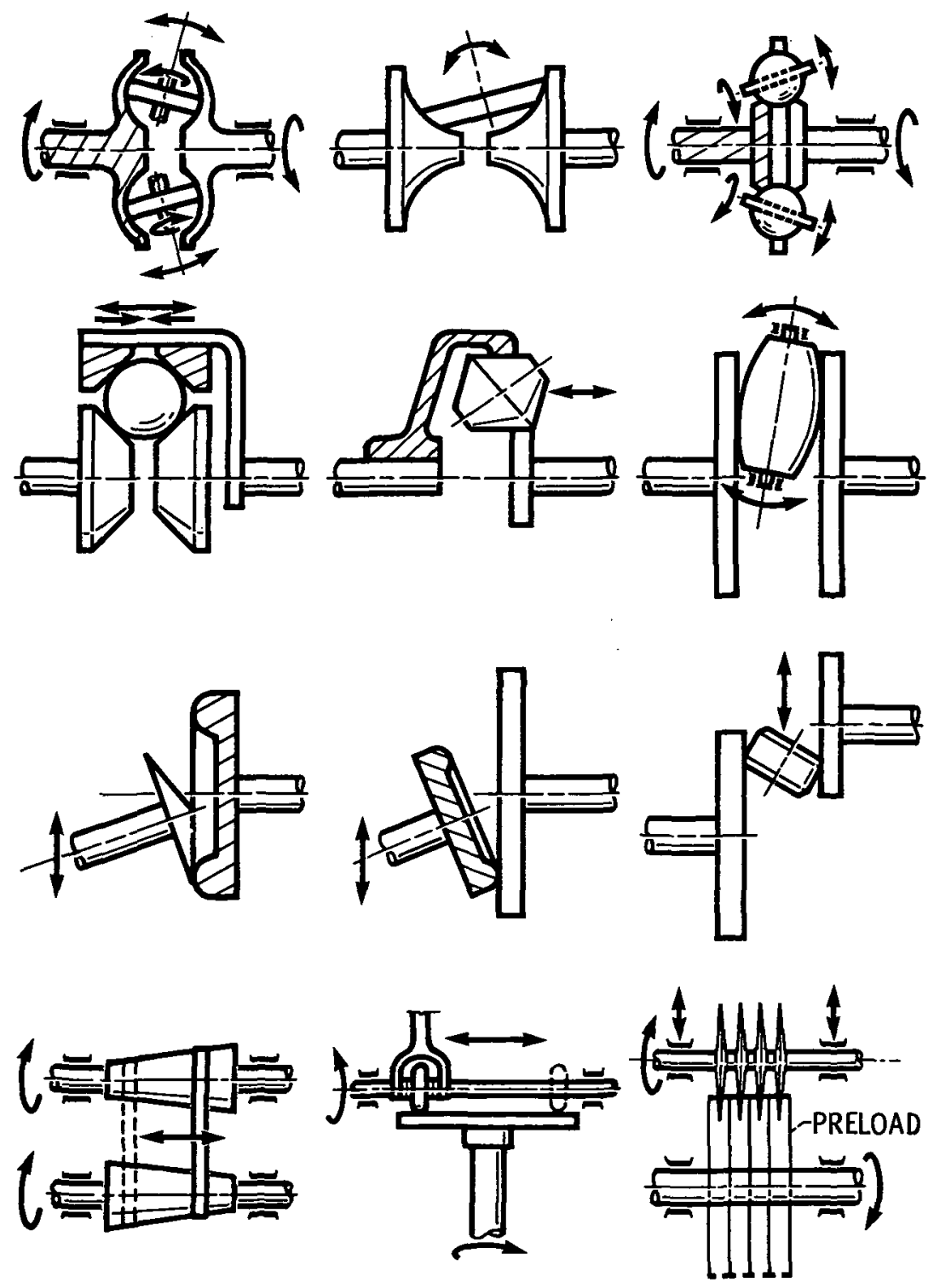

Figure 3. - Typical adjustable speed traction drive geometries. 


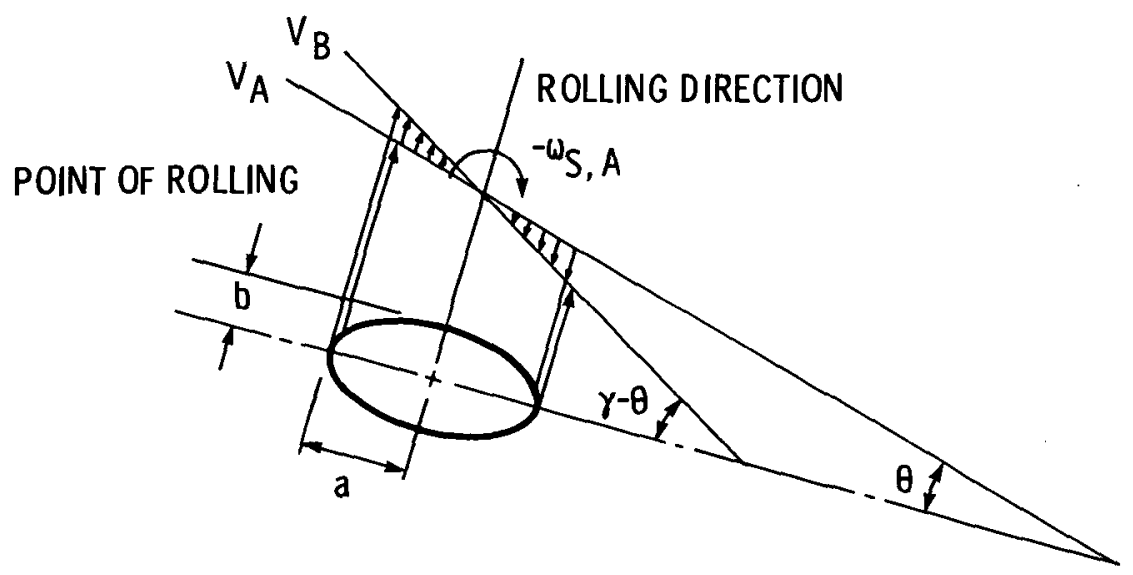

(a) Spin contact pattern on roller A.

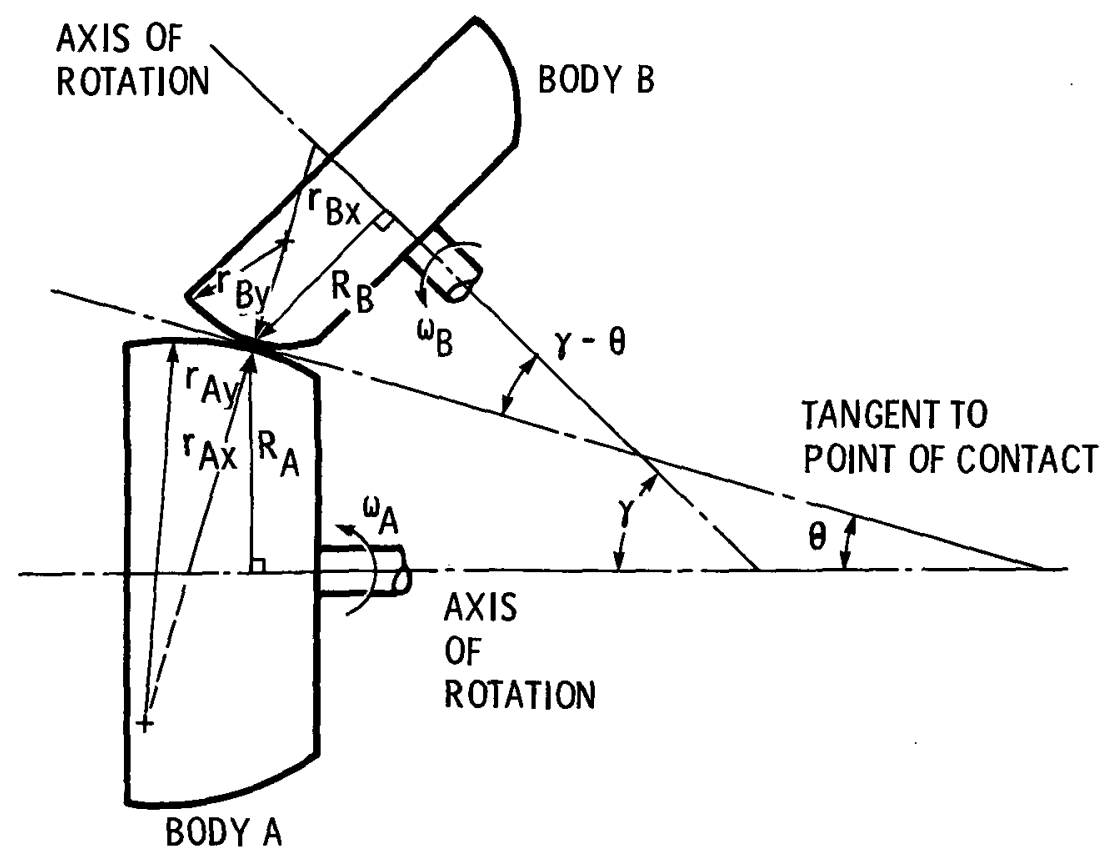

(b) Contact geometry for spin calculations.

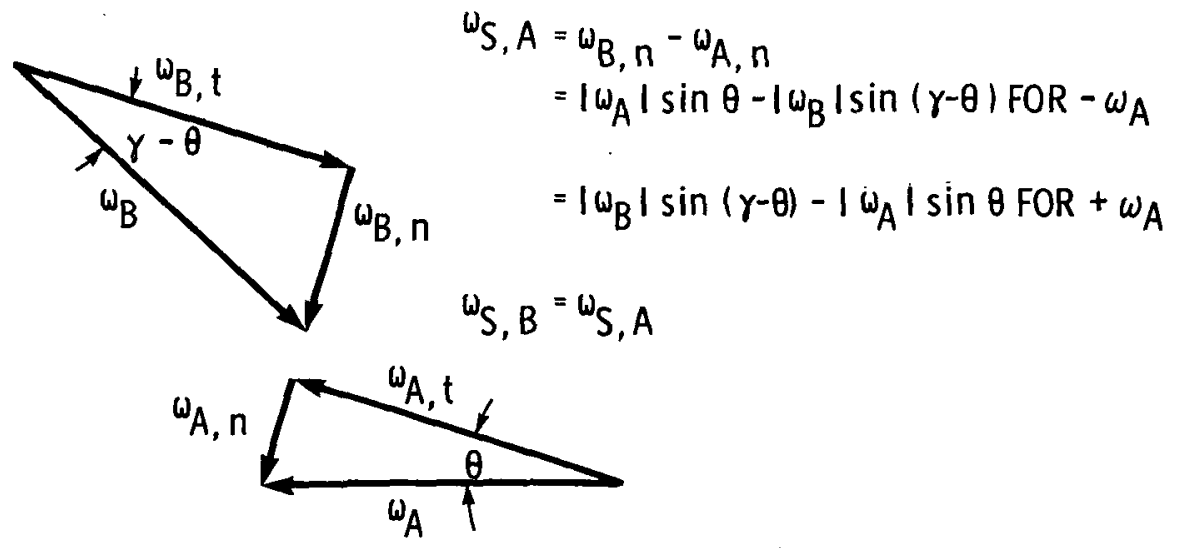

(c) Angular velocity diagram.

Figure 4. - Generalized velocity diagram for spin without applied traction. 

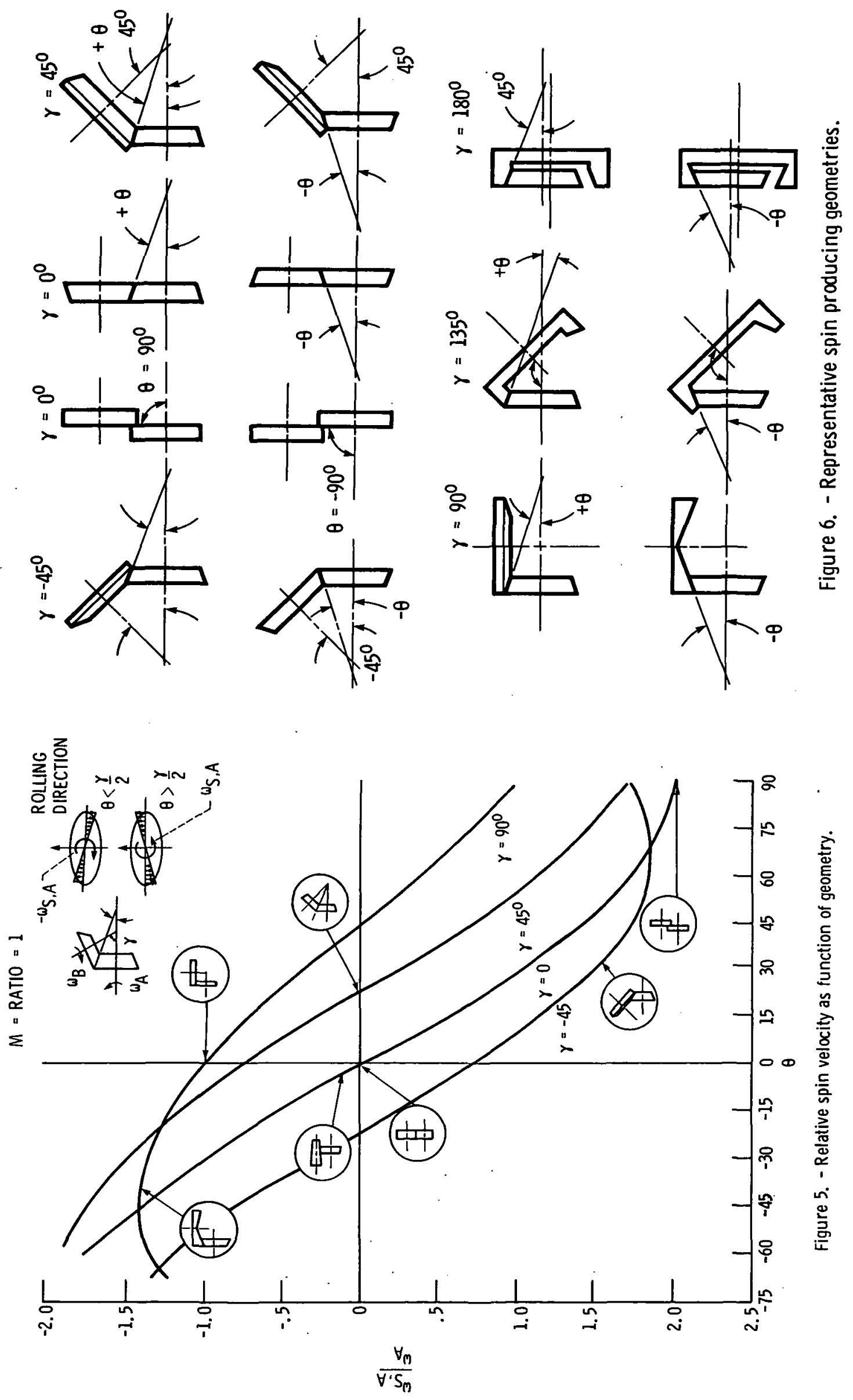

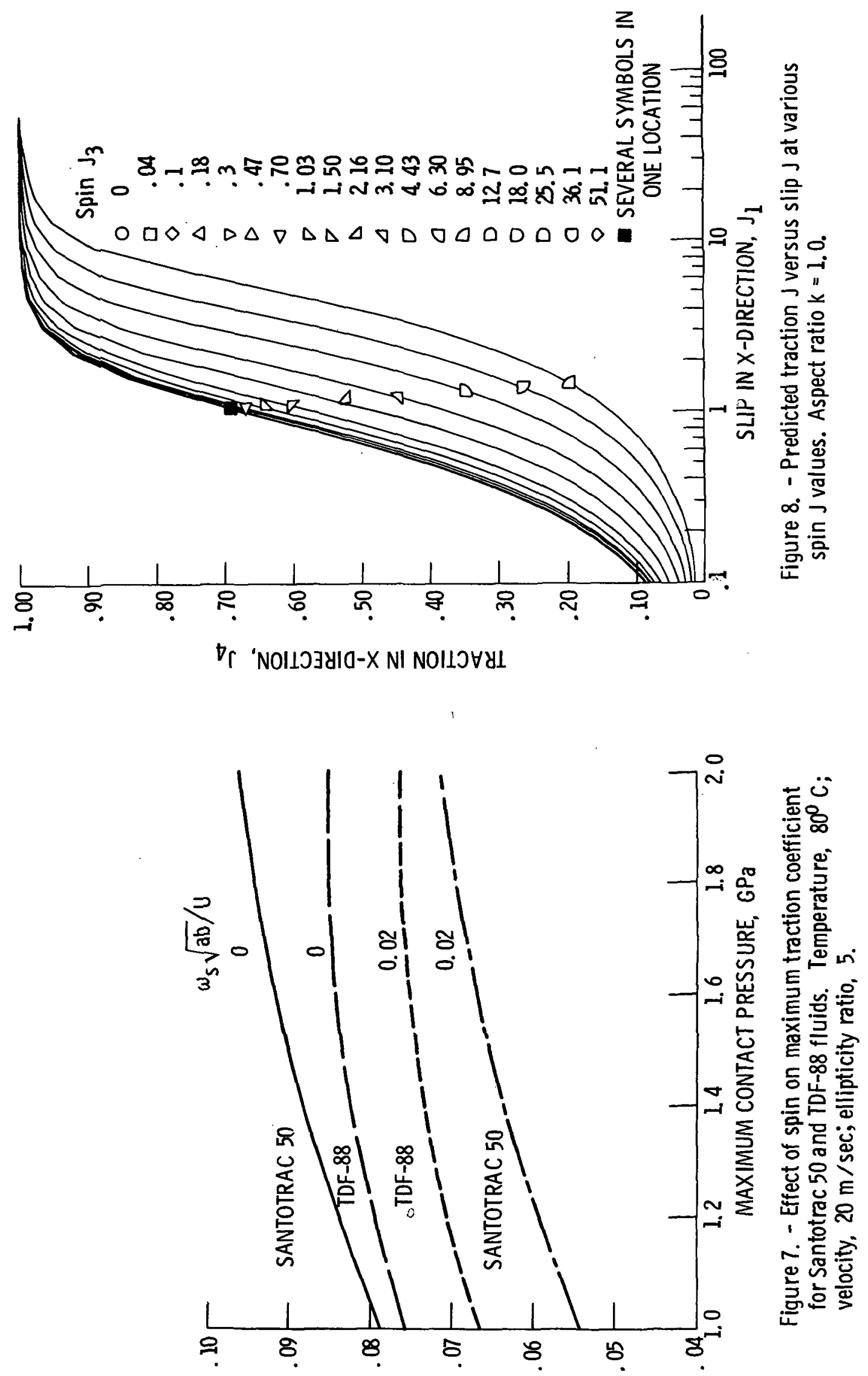


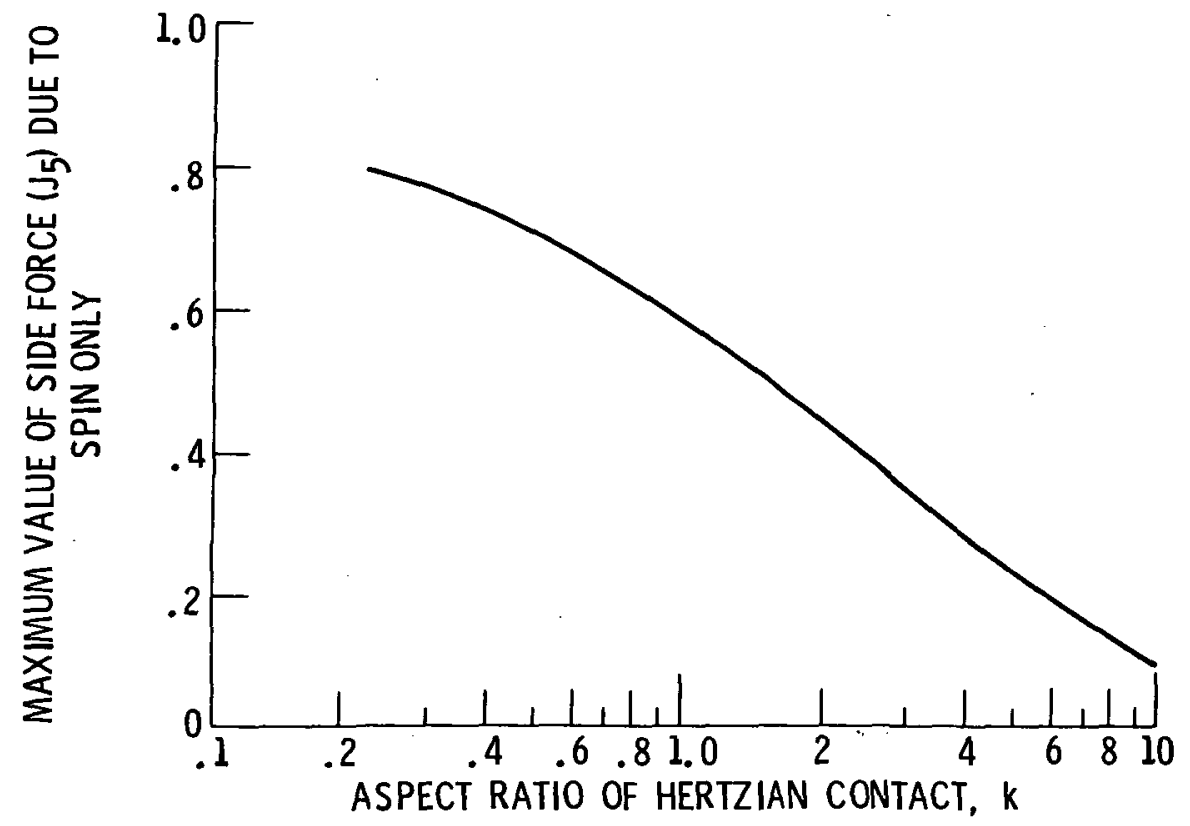

Figure 9. - Variation of maximum side force $\left(\mathrm{J}_{5}\right)$ with aspect ratio for contact under spin only. 


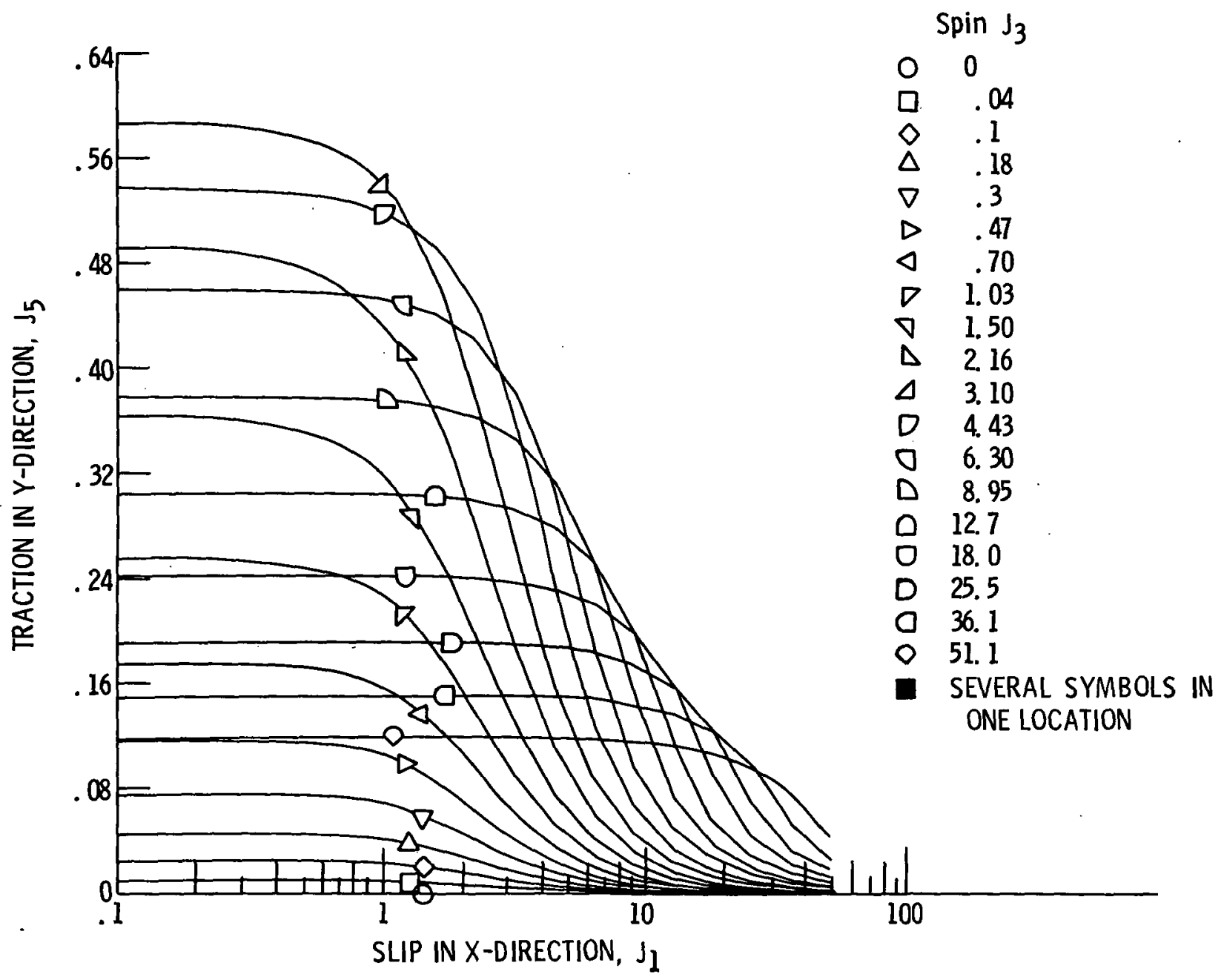

Figure 10. - Traction $J_{5}$ versus slip $J_{1}$ at various values of spin $J_{3}$. Aspect ratio of Hertzian contact $\mathrm{k}=1.00$. 


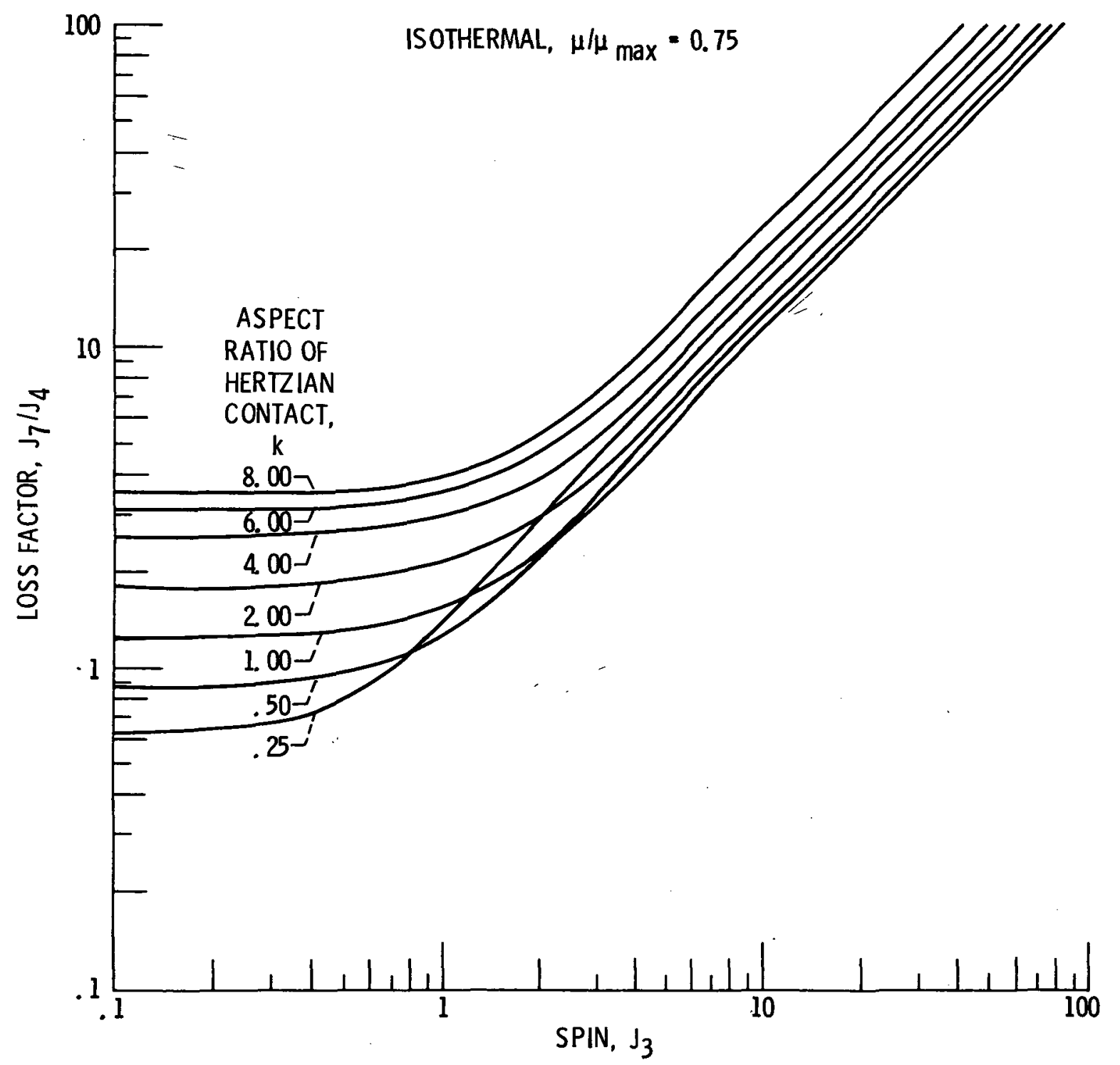

Figure 11. - Effect of spin and aspect ratio on predicted loss. 

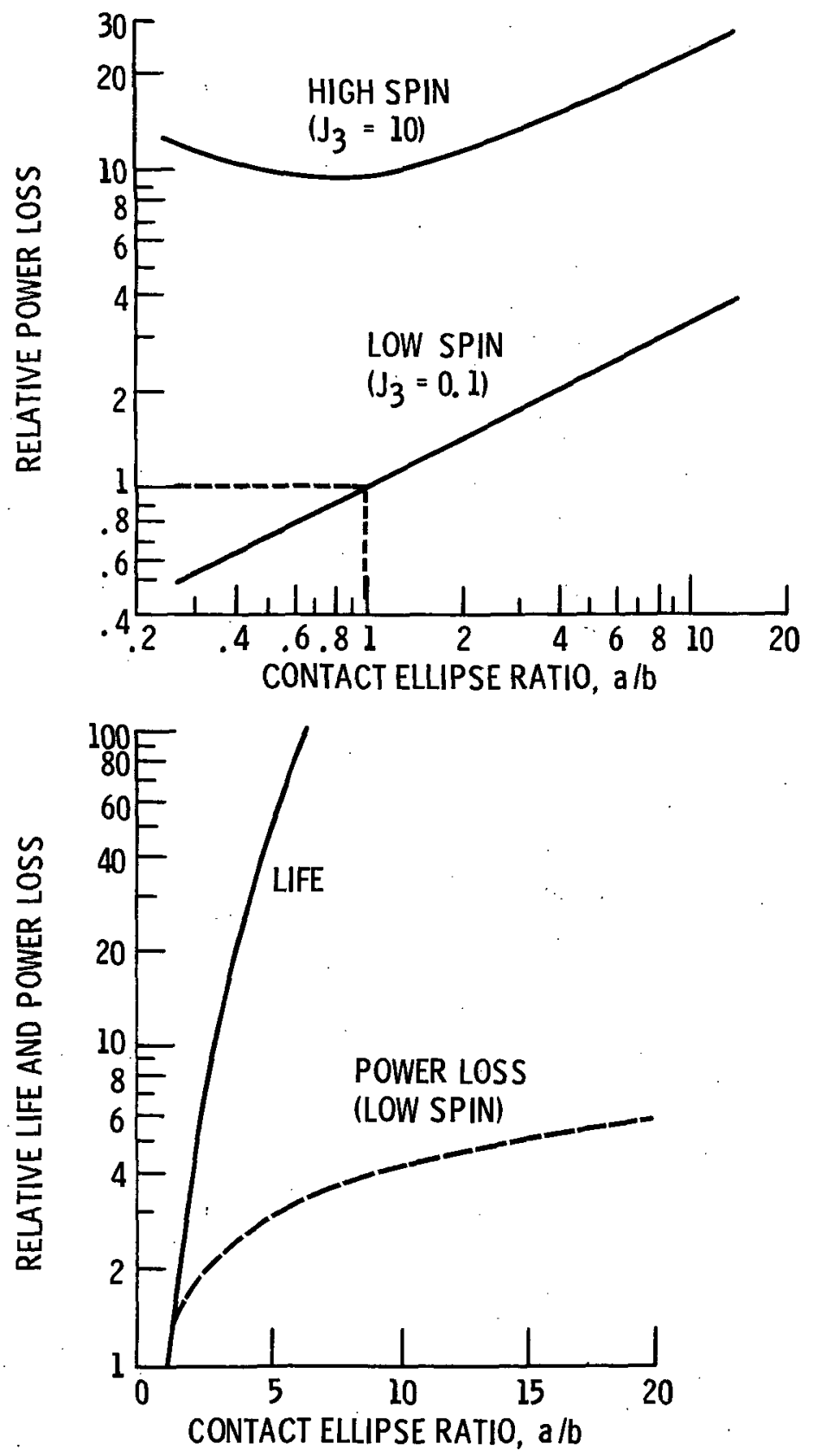

Figure 12. - Effect of aspect ratio on relative life and power loss. 


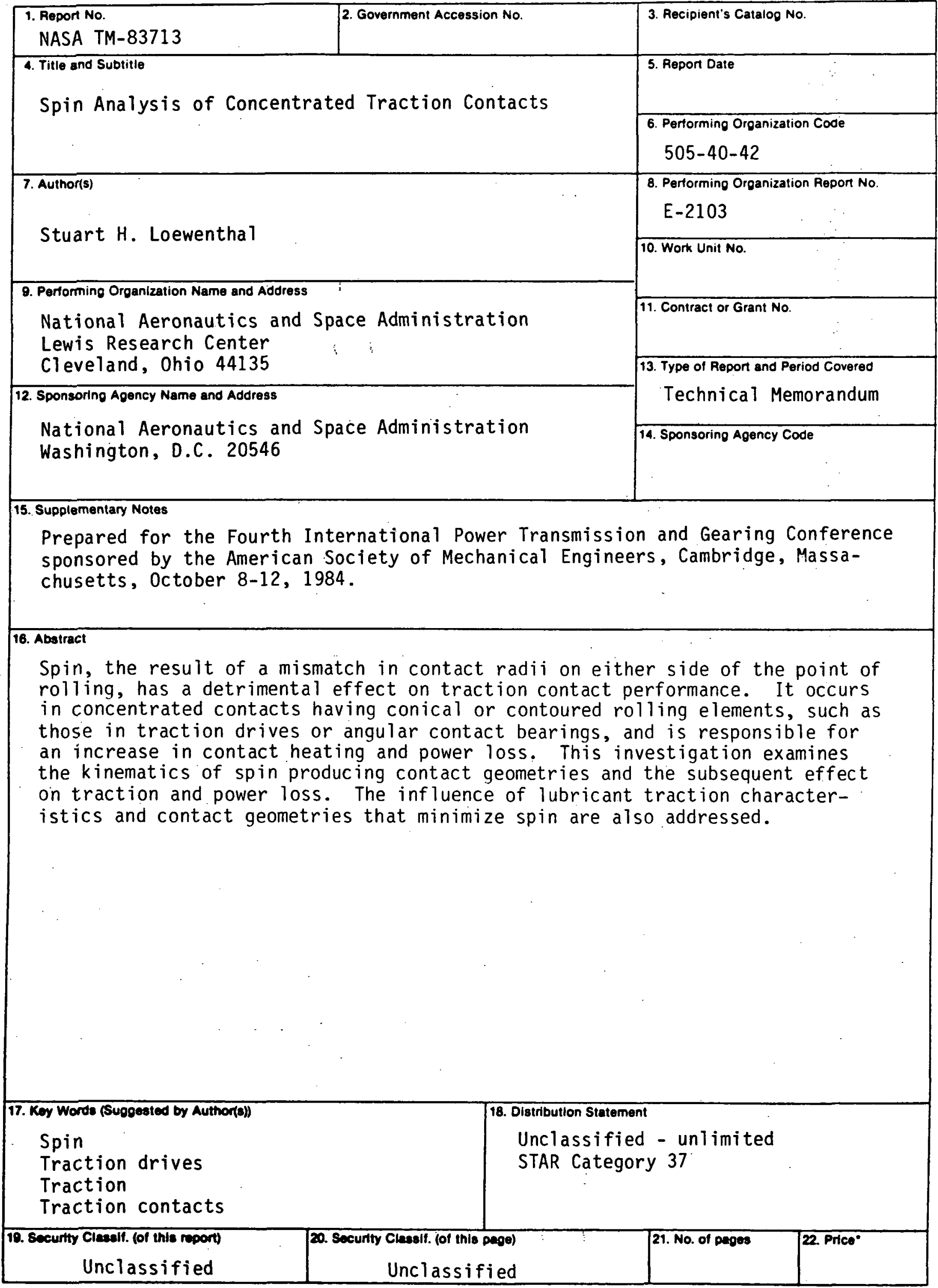


National Aeronautics and

Space Administration

Washington, D.C.

20546

Otficiel Baxines

Pendity for trimet Un, 100

Postege and Fees Paid Somen Administration unsa-451 